\title{
Fast molecular jet from L1157-mm ${ }^{\star, \star \star}$
}

\author{
M. Tafalla ${ }^{1}$, R. Bachiller ${ }^{1}$, B. Lefloch ${ }^{2,3}$, N. Rodríguez-Fernández ${ }^{4}$, C. Codella ${ }^{5}$, A. López-Sepulcre ${ }^{2,3,6}$, and L. Podio ${ }^{5}$ \\ 1 Observatorio Astronómico Nacional (IGN), Alfonso XII 3, 28014 Madrid, Spain \\ e-mail: m.tafalla@oan.es \\ 2 Univ. Grenoble Alpes, IPAG, 38000 Grenoble, France \\ 3 CNRS, IPAG, 38000 Grenoble, France \\ 4 CESBIO (UMR 5126), Obs. de Midi-Pyrénées (CNES, CNRS, Univ. Paul Sabatier, IRD), 31401 Toulouse, France \\ 5 INAF, Osservatorio Astrofisico di Arcetri, Largo E. Fermi 5, 50125 Firenze, Italy \\ ${ }^{6}$ Department of Physics, The University of Tokyo, Bunkyo-ku, 113-0033 Tokyo, Japan
}

Received 31 October 2014 / Accepted 25 November 2014

\section{ABSTRACT}

\begin{abstract}
Context. L1157-mm powers a molecular outflow that is well-known for its shock-induced chemical activity in several hot-spots. Aims. We have studied the molecular emission toward L1157-mm searching for a jet component responsible for these spots. Methods. We used the IRAM $30 \mathrm{~m}$ telescope to observe the vicinity of L1157-mm in several lines of SiO.

Results. The $\mathrm{SiO}(5-4)$ and $\mathrm{SiO}(6-5)$ spectra toward L1157-mm present blue and red detached components about $45 \mathrm{~km} \mathrm{~s}^{-1}$ away from the ambient cloud. These extremely high-velocity (EHV) components are similar to those found in the L1448 and IRAS $04166+2706$ outflows and probably arise from a molecular jet driven by L1157-mm. Observations of off-center positions indicate that the jet is unresolved in $\mathrm{SiO}(5-4)\left(<11^{\prime \prime}\right)$.

Conclusions. The EHV jet seen in SiO probably excites L1157-B1 and the other chemically active spots of the L1157 outflow.
\end{abstract}

Key words. stars: formation - ISM: abundances - ISM: jets and outflows - ISM: molecules

\section{Introduction}

The L1157 outflow was first identified by its unusual heating of the ambient gas toward the so-called B1 position; this is suggestive of a strong shock interaction (Umemoto et al. 1992). Many observations have confirmed this result and showed that molecular abundances in L1157-B1 and other hot-spots are enhanced by orders of magnitude in a manner consistent with models of shock chemistry (Mikami et al. 1992; Bachiller \& Pérez Gutiérrez 1997). The richness and intensity of the molecular spectrum from L1157-B1 has made this position a testbed for shock-chemistry models and a favored target for molecular surveys (Codella et al. 2010; Lefloch et al. 2010; Yamaguchi et al. 2012).

The spectra from L1157 (like those from other outflows) present broad wings. This means that most of the emitting gas moves with the lowest outflow velocities and that only a small fraction of the gas reaches the highest speeds. This distribution indicates that the emitting gas consists of accelerated ambient material and that the accelerating agent remains hidden.

A number of observations suggest that the exciting agent of the L1157 hot-spots is a jet. At high angular resolution, the emission from L1157-B1 resembles a bow shock whose vertex points away from the exciting source (Tafalla \& Bachiller 1995; Gueth et al. 1996), while high-excitation lines trace the reverse shock (Benedettini et al. 2012). In addition, the blue and red hotspots occur in pairs located symmetrically from the L1157-mm central source, and they seem to trace a slowly precessing jet

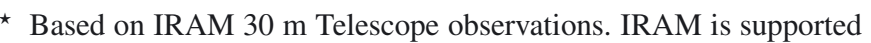
by INSU/CNRS (France), MPG (Germany), and IGN (Spain).

$\star \star$ Tables 1 and 2 are available in electronic form at http://www . aanda.org
}

(Zhang et al. 2000; Bachiller et al. 2001). Despite these indications, however, no direct detection of a jet component such as that seen in the L1448 and IRAS 04166+2706 outflows has been presented for L1157. Kristensen et al. (2012) have recently identified a red "bullet" in the water spectrum of L1157-mm. This feature could correspond to a true jet or to some other type of shock near the protostar. In this paper, we present $\mathrm{SiO}$ observations of the L1157-mm vicinity that convincingly show that a molecular jet is at the base of the L1157 outflow.

\section{Observations}

Our main dataset is a line survey toward L1157-mm carried out as part of the Astrochemical Surveys At IRAM (ASAI) project (Lefloch \& Bachiller, in prep.). This project covered the 1, 2, and $3 \mathrm{~mm}$ frequency bands that are reachable with the IRAM $30 \mathrm{~m}$ telescope ${ }^{1}$. L1157-mm was observed in September 2012 and February 2013 using the EMIR receiver and the FTS spectrometer, which provided a frequency resolution of $200 \mathrm{kHz}$. Wobblerswitching by $180^{\prime \prime}$ was used to ensure flat baselines, and each frequency setting consisted of two tunings $50 \mathrm{MHz}$ apart to separate lines coming from the upper and lower sidebands.

Follow-up $\mathrm{SiO}$ observations were carried out with the $30 \mathrm{~m}$ telescope in January-February and March 2014 using a setting similar to the ASAI project. They included integrations on L1448 mm and IRAS 04166+2706 (IRAS 04166 hereafter). Additionally, we here used $30 \mathrm{~m}$ data from two deep $\mathrm{CO}(2-1)$ integrations carried out in February 2001, also with similar settings.

The ASAI and 2014 data were compared and combined. The intensity of $\mathrm{SiO}(6-5)$ in these two datasets differed by a factor

1 http://www . oan.es/asai/ 


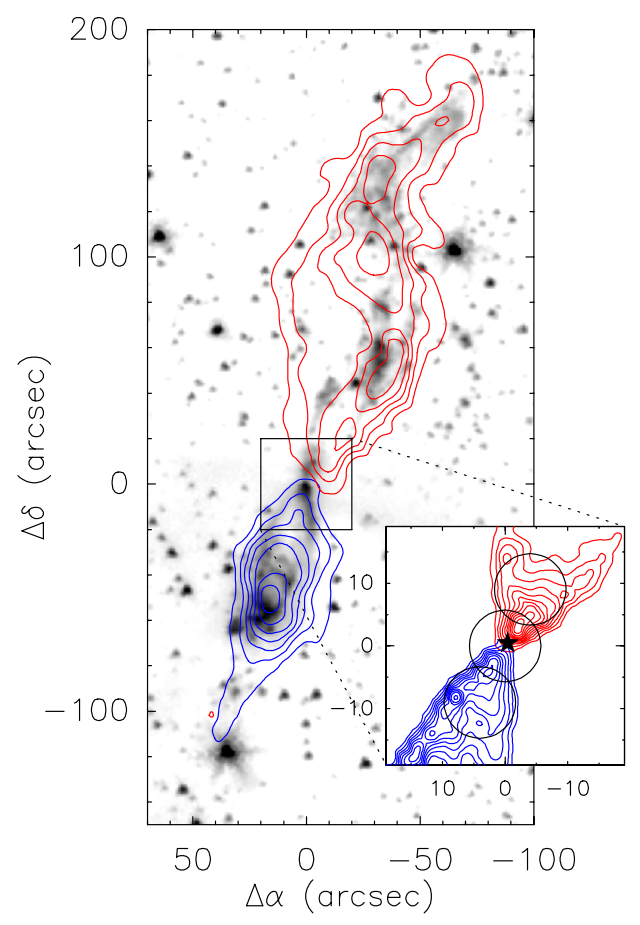

Fig. 1. Large-scale view of the L1157 outflow. The main frame shows a superposition of the CO map from Bachiller et al. (2001) (contours) and the $3.6 \mu \mathrm{m}$ Spitzer image of Looney et al. (2007). The inset shows the inner outflow according to the interferometric CO map of Jørgensen et al. (2007). The star symbol represents L1157-mm and the three circles represent the positions and beam sizes of our $\mathrm{SiO}(5-4)$ observations.

of 1.5 of unclear origin, and to avoid any bias, the data were averaged with equal weight. Standard efficiency values were applied to convert the data into to the $T_{\mathrm{mb}}$ scale used in the paper. A summary of the observations is presented in Tables 1 and 2.

\section{Spectral evidence for a jet in L1157-mm}

Figure 1 illustrates the geometry of the L1157 outflow with a superposition of a CO map (from Bachiller et al. 2001) and a $3.6 \mu \mathrm{m}$ Spitzer image (from Looney et al. 2007). An inset shows a high-resolution CO map toward the vicinity of the young stellar object (YSO, adapted from Jørgensen et al. 2007; see also Hull et al. 2014). This inner image reveals two opposed conical shells with the protostar located at their apex. The three circles mark the positions of the $\mathrm{SiO}(5-4)$ observations along the outflow discussed in Sect. 4.

Figure 2 shows the $\mathrm{SiO}(2-1),(5-4)$ and (6-5) spectra toward L1157-mm (the noisier $\mathrm{SiO}(3-2)$ ASAI spectrum is not shown). $\mathrm{The} \mathrm{SiO}(2-1)$ emission in the top panel consists of a narrow feature at the cloud velocity $\left(2.7 \mathrm{~km} \mathrm{~s}^{-1}\right.$, Bachiller et al. 2001) and low-intensity outflow wings. In contrast, the $\mathrm{SiO}(5-4)$ and (6-5) spectra (middle and bottom panels) show progressively weaker ambient features and increasingly more prominent blue and red detached components.

A comparison between the two ASAI spectra obtained with receiver tunings separated by $50 \mathrm{MHz}$ rules out contamination by lines in the image sideband as a possible origin of the detached components. In addition, a search in the JPL and CDMS spectroscopy databases (Pickett et al. 1998; Müller et al. 2001) shows no alternative molecular lines at the corresponding frequencies. The detached components must therefore arise from extremely high-velocity (EHV) SiO emission in the outflow. Previous evidence of EHV gas in L1157-mm was provided

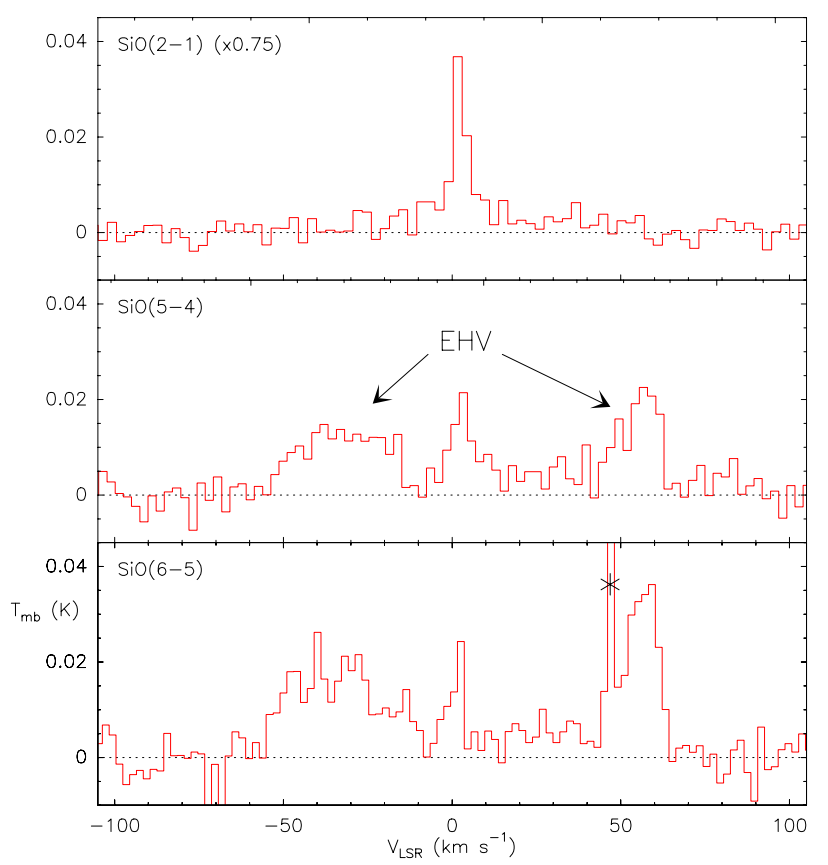

Fig. 2. $\mathrm{SiO}$ spectra toward L1157 $\mathrm{mm}\left(\mathrm{RA}(\mathrm{J} 2000)=20^{\mathrm{h}} 39^{\mathrm{m}} 06^{\mathrm{s}} .3\right.$, $\left.\operatorname{Dec}(\mathrm{J} 2000)=68^{\circ} 02^{\prime} 15^{\prime \prime} .8\right)$. The component near $2.7 \mathrm{~km} \mathrm{~s}^{-1}$, most prominent in $\mathrm{SiO}(2-1)$, arises from accelerated ambient gas, while the two components labeled "EHV" probably represent a molecular jet. An asterisk in the $\mathrm{SiO}(6-5)$ spectrum marks the contribution of ambient $c-\mathrm{C}_{3} \mathrm{H}_{2}\left(5_{32}-4_{41}\right)$.

by Kristensen et al. (2012), who identified a weak red "bullet" in the $\mathrm{H}_{2} \mathrm{O}\left(1_{10}-1_{01}\right)$ line. Our new data confirm this $\mathrm{H}_{2} \mathrm{O}$ detection, although the red $\mathrm{SiO}$ EHV peak is $10 \mathrm{~km} \mathrm{~s}^{-1}$ faster and 1.5 times narrower than the $\mathrm{H}_{2} \mathrm{O}$ peak. More importantly, the new $\mathrm{SiO}$ data show an equivalent $\mathrm{EHV}$ feature at blue velocities. While similar, the blue and red EHV components are not symmetric. Both $\mathrm{SiO}(5-4)$ and $\mathrm{SiO}(6-5)$ spectra show that the red EHV component is faster and narrower than the blue component, which suggests that this reflects an intrinsic property of the system.

Figure 3 compares the $\mathrm{SiO}(5-4)$ spectrum from L1157-mm with equivalent spectra from L1448 and IRAS 04166. It shows that despite differences in intensity of up to a factor of 20 , all EHV components have velocities of about $40-50 \mathrm{~km} \mathrm{~s}^{-1}$ with respect to the ambient cloud, are broad $\left(\sim 10 \mathrm{~km} \mathrm{~s}^{-1}\right)$, and often present asymmetric profiles with a flatter slope toward ambient velocities and a steeper drop toward higher speeds. The degree of symmetry between the blue and red EHV components varies over the sample, with L1157-mm presenting the two components with the most similar intensity (difference less than 40\%).

When the EHV emission of the L1448 and IRAS 04166 outflows has been observed with high angular resolution, it has been found to arise from a distinct jet-like component (Guilloteau et al. 1992; Santiago-García et al. 2009; Hirano et al. 2010). This component moves along the axis of the outflow cavity delineated by the lower-velocity gas and traces a series of internal working surfaces in a molecular jet (Santiago-García et al. 2009). The finding of an EHV component toward L1157-mm therefore reveals a fast molecular jet in the L1157 outflow.

\section{Extent of the EHV emission}

In the L1448 and IRAS 04166 outflows, the EHV emission is as extended as the low-velocity CO. In L1157, the emission is very compact. This is illustrated in Fig. 4 with an intensity cut along 


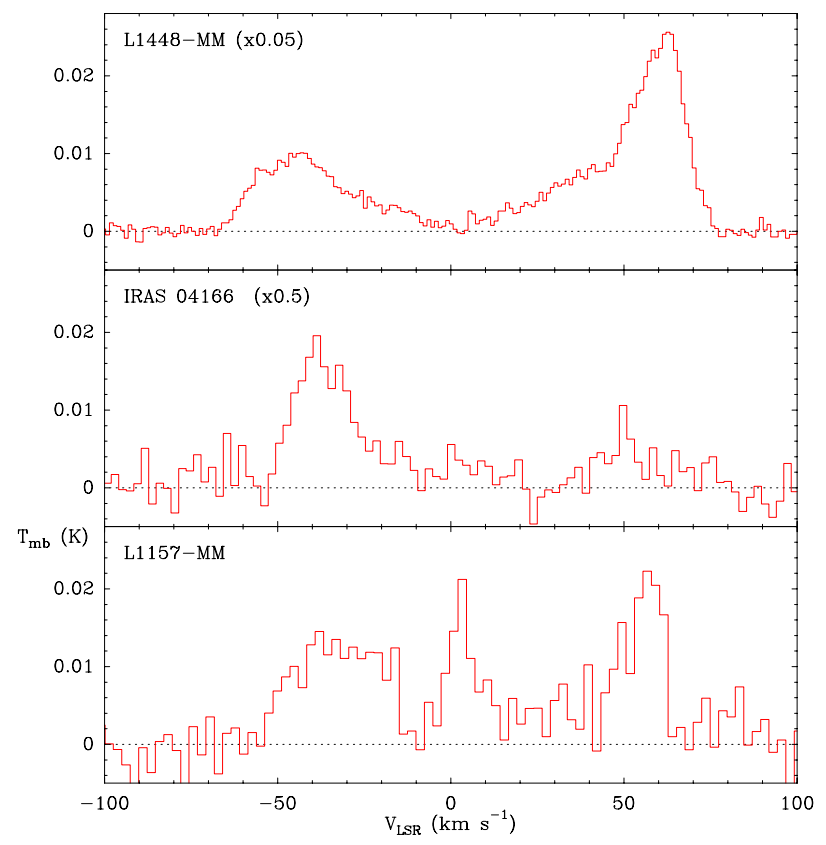

Fig. 3. $\mathrm{SiO}(5-4)$ spectra toward the outflow sources L1448-mm, IRAS 04166, and L1157-mm. All sources present EHV components of similar velocity but different intensity.

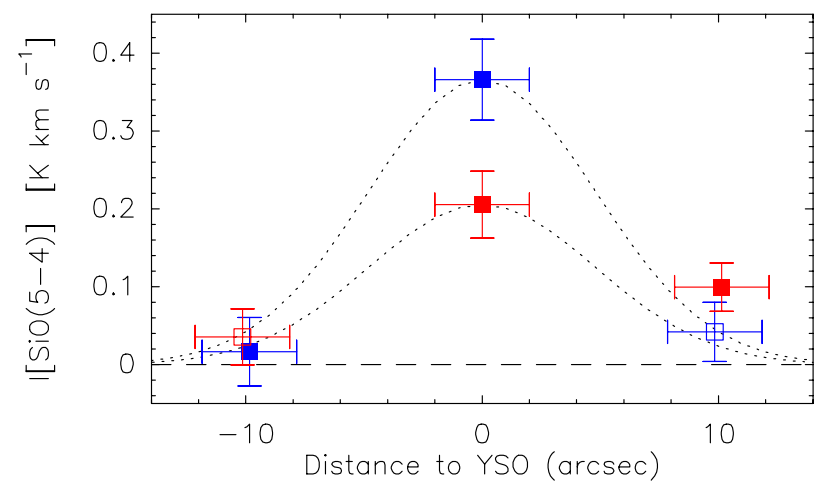

Fig. 4. Integrated intensities of the $\mathrm{SiO}$ (5-4) EHV emission (both blue and red components) measured at three positions along the L1157 outflow axis. The negative and positive distances correspond to the blue and red outflow lobes. The dotted curves are Gaussians with the size of the telescope beam at the $\mathrm{SiO}(5-4)$ frequency. A nominal rms position uncertainty of $2^{\prime \prime}$ has been assumed for all positions.

the axis of the outflow cavity. As a result of the weak $\mathrm{SiO}$ lines, which require several hours of integration, our observations of the EHV component in L1157 were limited to the three points indicated in Fig. 1, whose positions are given in Table 2. Even with this limited dataset, it is possible to constrain the emission size.

As Fig. 4 shows, no significant detection of EHV emission was obtained outside the central source. To model this result, we compared the data from each EHV component with a Gaussian that has the FWHM of the telescope beam at the $\mathrm{SiO}(5-4)$ frequency, which represents the expected intensity distribution for a point-like source centered on L1157- $\mathrm{mm}$. The dotted lines in Fig. 4 show that the blue EHV emission, which is expected to extend to negative distances, is consistent with being unresolved, while the red emission (expected toward positive distances) is at most marginally detected outside L1157-mm at a 3-sigma level. The size of the EHV emission must therefore be smaller than the $11^{\prime \prime}$ beam (a conversion to physical size depends on the uncertain source distance of 440-250 pc, Looney et al. 2007).

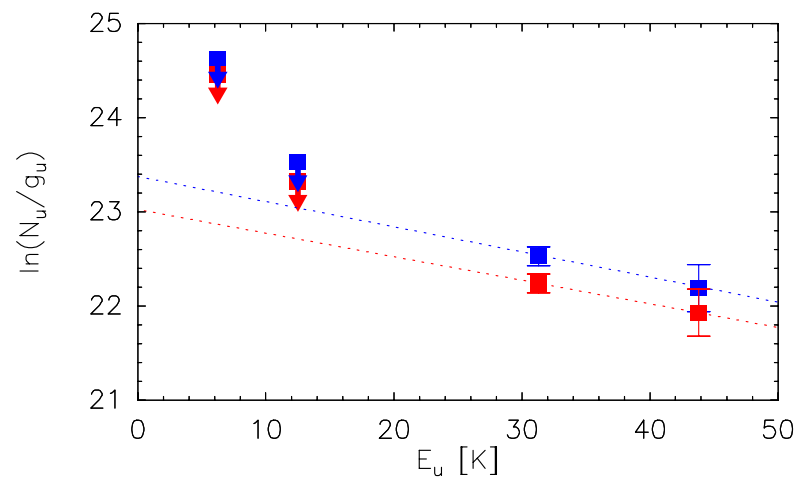

Fig. 5. Population diagram of the EHV SiO toward L1157-mm. The blue and red symbols represent blue and red EHV gas. The dashed lines represent two LTE fits consistent with the detections in $\mathrm{SiO}(5-4)$ and $\mathrm{SiO}(6-5)$ and with the 3-sigma upper limits in $\mathrm{SiO}(2-1)$ and $\mathrm{SiO}(3-2)$ (downward pointing arrows). See text for fit results.

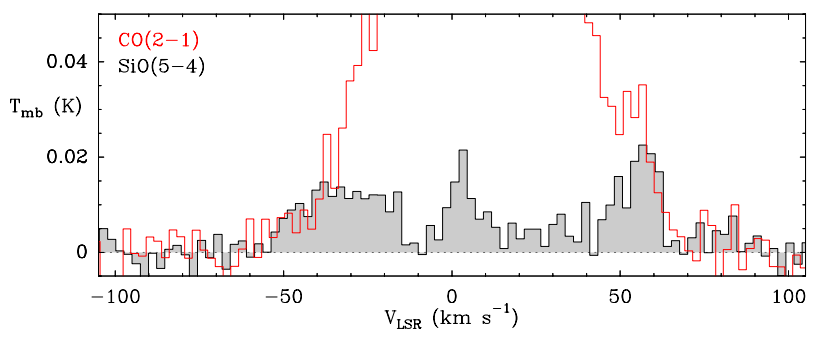

Fig. 6. Spectra of $\mathrm{CO}(2-1)$ (red) and $\mathrm{SiO}(5-4)$ (gray) toward L1157$\mathrm{mm}$. Note that the CO (2-1) line, which does not reveal distinct EHV emission, has similar velocity extents as the $\mathrm{SiO}(5-4)$ line with the same intensity level.

\section{SiO excitation, column density, and abundance}

After determining a limit to the size of the EHV emission, we now use the intensity of the different lines to estimate the excitation temperature and column density of $\mathrm{SiO}$ in the EHV gas. We assumed that the emitting region is unresolved and scaled all line intensities to a common size of $11^{\prime \prime}$ using point-source dilution factors. These scaled intensities were then converted into upper-level column densities assuming optically thin emission. The resulting population diagram is shown in Fig. 5.

Since the EHV component is only detected in $\mathrm{SiO}(5-4)$ and $\mathrm{SiO}(6-5)$, we used only these two lines to estimate the $\mathrm{SiO}$ excitation temperature and column density assuming LTE conditions. The error budget is dominated by calibration uncertainties, which we assumed are of $10 \%$ for $\mathrm{SiO}(5-4)$ and of $25 \%$ for $\mathrm{SiO}(6-5)$. With these values, we estimate that the blue and red EHV components have a similar $\mathrm{SiO}$ excitation temperature of $40 \pm 30 \mathrm{~K}$, while their $\mathrm{SiO}$ column densities are $(5 \pm 4) \times 10^{11}$ and $(4 \pm 3) \times 10^{11} \mathrm{~cm}^{-2}$. As shown by the dashed lines in Fig. 5, the fits are consistent with the nondetections of $\mathrm{SiO}(2-1)$ and $\mathrm{SiO}(3-2)$. Compared with similar estimates in the L1448 and IRAS 04166 outflows, the column densities in L1157-mm are lower by about an order of magnitude (Tafalla et al. 2010).

To estimate the $\mathrm{SiO}$ abundance in the EHV component, we used the intensity of $\mathrm{CO}(2-1)$, which was observed by the ASAI project with an angular resolution similar to that of $\mathrm{SiO}(5-4)$. The $\mathrm{CO}(2-1)$ spectrum is shown in Fig. 6 and differs from that of $\mathrm{SiO}(5-4)$ in that it has no distinct EHV components. Instead, it has broad outflow wings that extend as far as the EHV range (there is a hint of a red EHV peak at the noise level). The different shape of the CO and $\mathrm{SiO}$ spectra is also seen in L1448 and IRAS 04166 and seems to be caused by the $\mathrm{SiO}$ emission being more selective of the EHV component than the $\mathrm{CO}$ emission, 


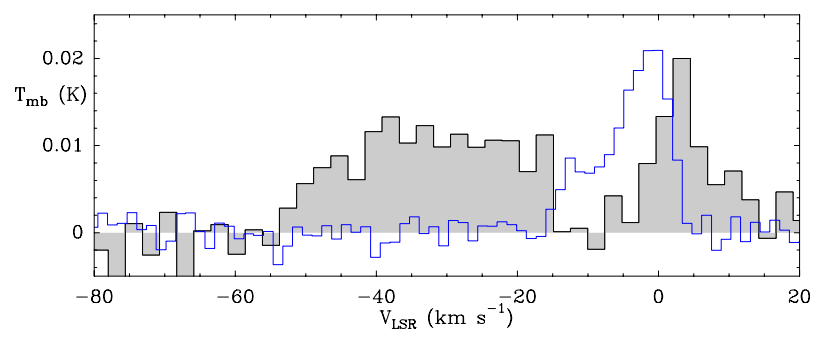

Fig. 7. $\mathrm{SiO}(5-4)$ spectra toward L1157-mm (gray) and L1157-B1 (blue). L1157-B1 spectrum scaled down by factor of 100 .

which traces both the EHV gas and the lower-velocity outflow. While it is not possible to separate the EHV and wing components in the $\mathrm{CO}$ spectrum, the plot shows that the intensities of $\mathrm{CO}(2-1)$ and $\mathrm{SiO}(5-4)$ are approximately equal at the velocities of the SiO EHV regime.

If we assume that the $\mathrm{CO}$ and $\mathrm{SiO}$ emissions are optically thin and have the same excitation temperature of $40 \mathrm{~K}$, the observed $\mathrm{CO}(2-1) / \mathrm{SiO}(5-4)$ intensity ratio of 1 implies a column density ratio of $\approx 500$. Assuming a $\mathrm{CO}$ abundance of $8.5 \times 10^{-5}$ (Frerking et al. 1982), we derive an $\mathrm{SiO}$ abundance of $\approx 1.7 \times 10^{-7}$. This value agrees within a factor of 2 with the abundance estimated by Tafalla et al. (2010) in the EHV component of the L1448 and IRAS 04166 outflows.

\section{Relation with L1157-B1}

The EHV SiO jet is the natural candidate for the exciting agent of L1157-B1 and the other outflow hot-spots. To explore this possibility, we compare in Fig. 7 the $\mathrm{SiO}(5-4)$ emissions from L1157$\mathrm{mm}$ and L1157-B1 (from Bachiller et al. 2001). As can be seen, the EHV component in L1157-mm is faster than the main emission in the L1157-B1 spectrum, as would be expected if the EHV gas is the driving agent. (The EHV component reaches higher velocities than the fastest CO gas detected in L1157-B1 to date, that is, the $g_{1}$ component of Lefloch et al. 2012, which is probably associated with the jet shock.) To be more quantitative, we estimate from Fig. 7 that the blue EHV component has a mean velocity of $35 \mathrm{~km} \mathrm{~s}^{-1}$ (from a Gaussian fit), and that the main $\mathrm{SiO}$ emission in L1157-B1 reaches a velocity of about $18 \mathrm{~km} \mathrm{~s}^{-1}$. If these two velocities correspond to the jet and bow shock components, their ratio approximately equals 2 . A simple requirement of momentum conservation, necessary for the jet to accelerate the shock, implies that the velocity ratio equals $1+\sqrt{\rho / \rho_{j}}$, where $\rho$ and $\rho_{j}$ are the ambient cloud and jet densities (see, e.g., Masson \& Chernin 1993). Both the ambient and jet densities are very uncertain, but they lie very probably in the vicinity of $10^{5} \mathrm{~cm}^{-3}$, which is typical of dense cores and molecular jets (Santiago-García et al. 2009). If so, their ratio should be close to 1 , and $v_{j} / v_{s}$ should be $\approx 2$, as observed.

To be the accelerating agent, in addition to having the correct velocity, the EHV gas needs to reach L1157-B1, which is more than $60^{\prime \prime}$ away from L1157-mm. The EHV SiO emission is detected only toward L1157-mm, but this likely results from a combination of limited sensitivity and an intensity drop of the $\mathrm{SiO}$ emission away from the central source similar to that seen in the L1448 and IRAS 04166 outflows (Santiago-García et al. 2009; Hirano et al. 2010). Thus, to confirm that the EHV gas reaches L1157-B1, we need to rely on the CO emission, which is less selective of the EHV gas due to the broad wing component. Unfortunately, no large-scale deep CO observations are available

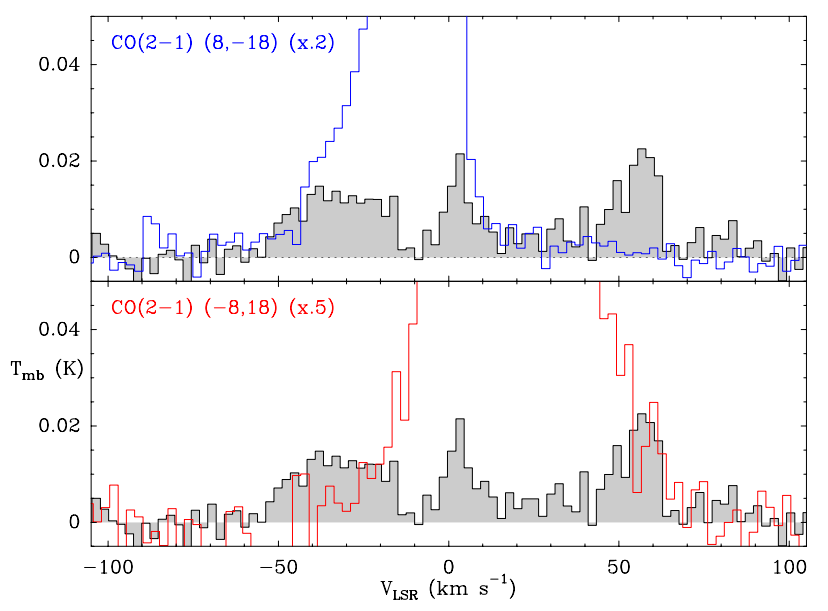

Fig. 8. On-source $\mathrm{SiO}(5-4)$ spectrum versus $\mathrm{CO}(2-1)$ spectra $20^{\prime \prime}$ from the source. In each panel, the $\mathrm{SiO}(5-4)$ spectrum toward L1157-mm is shown in gray and the $\mathrm{CO}(2-1)$ spectra at positions $20^{\prime \prime}$ away from L1157-mm are shown in color.

to follow the EHV emission between L1157-mm and L1157-B1. Our limited data do suggest that there is such an emission, however. This is illustrated Fig. 8 with $\mathrm{CO}(2-1)$ spectra toward two positions 20" away from L1157-mm in the direction of the expected jet. As can be seen, both blue and red CO spectra extend in velocity as far as the $\mathrm{SiO}$ EHV emission from L1157-mm, indicating that the EHV gas extends farther than indicated by the $\mathrm{SiO}$ emission. Thus, although further observations are needed to complete the picture, our data suggest that the exciting jet of the L1157 outflow has finally been identified.

Acknowledgements. We thank the IRAM staff for assistance and the referee for a careful reading of the manuscript. We acknowledge support from projects FIS2012-32096 and AYA2012-32032 of Spanish MINECO and from the MICINN program CONSOLIDER INGENIO 2010, grant "Molecular Astrophysics: The Herschel and ALMA era - ASTROMOL" (ref.: CSD200900038). LP has received funding from the European Union Seventh Framework Programme (FP7/2007-2013) under grant agreement No. 267251. This research has made use of NASA's Astrophysics Data System Bibliographic Services.

\section{References}

Bachiller, R., Pérez Gutiérrez, M., Kumar, M. S. N., \& Tafalla, M. 2001, A\&A, 372,899

Bachiller, R., \& Pérez Gutiérrez, M. 1997, ApJ, 487, L93

Benedettini, M., Busquet, G., Lefloch, B., et al. 2012, A\&A, 539, L3

Codella, C., Lefloch, B., Ceccarelli, C., et al. 2010, A\&A, 518, L112

Frerking, M. A., Langer, W. D., \& Wilson, R. W. 1982, ApJ, 262, 590

Gueth, F., Guilloteau, S., \& Bachiller, R. 1996, A\&A, 307, 891

Guilloteau, S., Bachiller, R., Fuente, A., \& Lucas, R. 1992, A\&A, 265, L49

Hirano, N., Ho, P. P. T., Liu, S.-Y., et al. 2010, ApJ, 717, 58

Hull, C. L. H., Plambeck, R. L., Kwon, W., et al. 2014, ApJS, 213, 13

Jørgensen, J. K., Bourke, T. L., Myers, P. C., et al. 2007, ApJ, 659, 479

Kristensen, L. E., van Dishoeck, E. F., Bergin, E. A., et al. 2012, A\&A, 542, A8

Lefloch, B., Cabrit, S., Codella, C., et al. 2010, A\&A, 518, L113

Lefloch, B., Cabrit, S., Busquet, G., et al. 2012, ApJ, 757, L25

Looney, L. W., Tobin, J. J., \& Kwon, W. 2007, ApJ, 670, L131

Masson, C. R., \& Chernin, L. M. 1993, ApJ, 414, 230

Mikami, H., Umemoto, T., Yamamoto, S., \& Saito, S. 1992, ApJ, 392, L87

Müller, H. S. P., Thorwirth, S., et al. 2001, A\&A, 370, L49

Pickett, H. M., Poynter, R. L., et al. 1998, J. Quant. Spectr. Rad. Transf., 60, 883

Santiago-García, J., Tafalla, M., et al. 2009, A\&A, 495, 169

Tafalla, M., \& Bachiller, R. 1995, ApJ, 443, L37

Tafalla, M., Santiago-García, J., et al. 2010, A\&A, 522, A91

Umemoto, T., Iwata, T., Fukui, Y., et al. 1992, ApJ, 392, L83

Yamaguchi, T., Takano, S., Watanabe, Y., et al. 2012, PASJ, 64, 105

Zhang, Q., Ho, P. T. P., \& Wright, M. C. H. 2000, AJ, 119, 1345

Page 5 is available in the electronic edition of the journal at http://www . aanda.org 
M. Tafalla et al.: Fast molecular jet from L1157-mm

Table 1. Transitions observed toward L1157-mm.

\begin{tabular}{lrrrr}
\hline \hline Transition & $\begin{array}{r}\text { Frequency } \\
(\mathrm{GHz})\end{array}$ & $\begin{array}{r}F W H M \\
\left({ }^{\prime \prime}\right)\end{array}$ & $\eta_{\mathrm{mb}}$ & $\begin{array}{r}\sigma\left(T_{\mathrm{mb}}\right)^{a} \\
(\mathrm{mK})\end{array}$ \\
\hline $\mathrm{SiO}(2-1)$ & 86.8 & 28 & 0.81 & 3.8 \\
$\mathrm{SiO}(3-2)$ & 130.3 & 19 & 0.76 & 6.2 \\
$\mathrm{SiO}(5-4)$ & 217.1 & 11 & 0.62 & 3.3 \\
$\mathrm{SiO}(6-5)$ & 260.5 & 9 & 0.53 & 4.8 \\
\hline
\end{tabular}

Notes. ${ }^{(a)}$ In a $1 \mathrm{~km} \mathrm{~s}^{-1}$ channel.
Table 2. Additional observations toward the L1157 outflow.

\begin{tabular}{lrrr}
\hline \hline Transition & $\begin{array}{r}\text { Frequency } \\
(\mathrm{GHz})\end{array}$ & $\begin{array}{r}F W H M \\
\left({ }^{\prime \prime}\right)\end{array}$ & $\begin{array}{r}\text { Offsets }^{a} \\
\left({ }^{\prime \prime},{ }^{\prime \prime}\right)\end{array}$ \\
\hline $\mathrm{SiO}(5-4)$ & 217.1 & 11 & $(-4,9),(4,-9)$ \\
$\mathrm{CO}(2-1)$ & 230.5 & 11 & $(0,0),(-8,18),(8,-18)$ \\
\hline
\end{tabular}

Notes. ${ }^{(a)}$ Measured from RA $(\mathrm{J} 2000)=20^{\mathrm{h}} 39^{\mathrm{m}} 06 \mathrm{~s} 3, \operatorname{Dec}(\mathrm{J} 2000)=$ $68^{\circ} 02^{\prime} 15^{\prime \prime} 8$ 